\title{
Three-dimensional ultrasonographic evaluation of carotid artery plaque surface irregularity
}

Hakan Artas, Izzet Okcesiz

Department of Radiology, Faculty of Medicine, Firat University, Elazığ, Turkey

Submitted: 12 October 2018

Accepted: 30 November 2018

Arch Med Sci 2020; 16 (1): 58-65

DOI: https://doi.org/10.5114/aoms.2018.81135

Copyright @ 2018 Termedia \& Banach

\section{Abstract}

Introduction: The aim of this study was to evaluate 3-dimensional (3D) ultrasonography (US) in determining the surface irregularity of carotid artery plaques.

Material and methods: This study included 50 patients (20 females and 30 males) aged between 56 and 82 years with plaques in the carotid artery which were detected during routine neck ultrasound. Simultaneously these cases were evaluated in terms of plaque echogenicities and surface characteristics with 2D and 3D US.

Results: 3D imaging was successfully performed in 45 of the 50 cases and the technical success rate was $90 \%$. A single plaque was detected in $64.4 \%$ of the patients, with the remaining $35.6 \%$ having more than one plaque. The lengths of the plaques ranged from 2 to $12 \mathrm{~mm}$ (mean: $3.98 \pm 1.70 \mathrm{~mm}$ ); the widths ranged from 1.8 to $3.2 \mathrm{~mm}$ (mean: $2.11 \pm 0.37 \mathrm{~mm}$ ). No significant difference was found between $2 \mathrm{D}$ and $3 \mathrm{D}$ plaque echo-structures (observer 1 , $p=0.317$; observer $2, p=0.276$ ), but there were significant differences between 2D and 3D plaque surface irregularities (observer 1, $p=0.002$; observer $2, p=0.004$ ). The inter-observer agreement on $2 \mathrm{D}$ and $3 \mathrm{D}$ plaque echo-structure and surface irregularity was very good ( $\kappa$ coefficients were 0.89 and 0.83 , respectively, for echo-structure, and 0.91 and 0.95 , respectively, for surface irregularity).

Conclusions: The present study shows that 3D US examination is a valuable non-invasive method for investigation of surface irregularity of carotid artery plaques.

Key words: carotid plaque, 3D ultrasonography, surface irregularity.

\section{Introduction}

Carotid artery disease is one of the main causes of ischemic cerebrovascular diseases (CVDs). The degree of carotid artery stenosis and ulcerated plaques are known as the major risk factors of ipsilateral CVDs [1, 2]. The North American Symptomatic Carotid Endarterectomy Trial (NASCET) and European Carotid Surgery Trial (ECST) trialists found that when carotid plaque surface contour irregularities, primarily ulceration, were seen on angiograms in patients with symptoms, the risk of CVDs was substantially higher than when a smooth plaque surface was suggested by the angiogram [1, 3]. Recent randomized clinical trials report that performing carotid endarterectomy and carotid stenting operations on symptomatic or asymptomatic patients with carotid artery stenosis significantly decreases the risk of ipsilateral CVDs [4-6]. Carotid plaque with an irregular

\author{
Corresponding author: \\ Hakan Artas MD \\ Department \\ of Radiology \\ Faculty of Medicine \\ Firat University \\ Elazığ, Turkey \\ Phone: +90 4242333555 \\ E-mail: hakanartas@ \\ yahoo.com
}


surface increased the risk of ischemic CVDs threefold and the cumulative 5-year ischemic CVD risk among individuals with an irregular plaque surface was over $8 \%$, whereas those with regular plaque had a 3\% risk over 5 years [7].

Technologic innovations will likely improve carotid artery imaging ability. The choice of imaging strategy is also important in asymptomatic carotid artery disease. The fundamental goal in carotid imaging is to provide better risk stratification for patients with carotid atherosclerosis. Accurate assessment of carotid artery plaques is an important step in the treatment planning and the follow-up. In the past, conventional angiography was generally used to select patients for treatment [8]. However, given the risks of death and disabling stroke due to angiography $(1.2 \%$ in the Asymptomatic Carotid Atherosclerosis Study [9] vs. 1.1\% for surgery itself), alternative noninvasive imaging techniques have been sought and investigated during the last years $[8,9]$. The carotid bifurcation lies close to the surface of the neck, without overlying bony structures or air spaces. Transcutaneous ultrasound has been a very effective tool for evaluating carotid disease. Ultrasonography provides detailed information regarding carotid plaques such as the degree of stenosis, surface regularity, echo-structure and intima-media thickness [8].

Two-dimensional (2D) ultrasonography (US) has been useful in assessing carotid plaque [7]. The major limitation with 2D US is that it is an operator dependent method, relying on the information obtained from the operator's reconstruction of sectional plaque images. To avoid this limitation, three-dimensional (3D) US is available as an important screening method in carotid artery imaging. Notably, 3D US provides an overall image of all plaque sections on the same plane [10].

The aim of this study is to evaluate 3D US in determining the echo-structure and surface irregularity of carotid artery plaques.

\section{Material and methods}

A total of 50 neurologically asymptomatic patients (20 females and 30 males, aged between 56 and 82; mean age: $68.5 \pm 7.8$ ) who had carotid plaques that had been detected during routine neck ultrasound (such as thyroid disease, neck pain, etc.) were consecutively enrolled in this study.

Simultaneously 3D ultrasound was performed in these cases, and 2D and 3D US data were recorded. All plaques were evaluated for their echo-structure, surface regularity (smooth or irregular) and plaque size in $2 \mathrm{D}$ and 3D US images. The echo-structures of the plaques were classified as follows [11]: class I, homogeneous texture, uniformly hypoechoic echogenicity; class II, heterogeneous texture, predominantly hypoechoic echogenicity; class III, heterogeneous texture, predominantly hyperechoic echogenicity; class IV, homogeneous texture, uniformly hyperechoic echogenicity and class V, unclassified calcified plaques. Carotid plaque was defined as a localized echostructure that encroached into the vessel $>1 \mathrm{~mm}$ beyond the interface between the lumen and intima [12]. Surface characteristics were classified as smooth and irregular (height variations of $>0.4 \mathrm{~mm}$ [13].

Each patient gave his or her written informed consent. The approval of the ethics committee was obtained.

\section{Two- and three-dimensional ultrasonography examinations}

The same investigator performed the 2D and 3D US examinations (with a Toshiba Xario device (Tokyo, Japan), using a 7-12 MHz linear transducer). This investigator has 10 years of experience with 3D ultrasound and carotid US. At the beginning of the study, the common carotid artery, internal carotid artery and carotid bulb were scanned in multiple projections in longitudinal and transverse planes. Two-dimensional images were recorded at the level of the carotid plaque, where the plaque appeared largest, providing the highest quality images. Subsequently, 3D data in the longitudinal or transverse positions were obtained and recorded for further analysis, with freehand technique at this level. The investigator moved the transducer with a smooth, steady motion. The acquisitions in freehand technique were made more frequently longitudinally (in 40 cases $-88.9 \%$ ) than axially (in 5 cases $-11.1 \%$ ). In patients with short neck and high bifurcation, acquisitions were made axially. All data acquisitions were made with sweep movement techniques. After scanning, the software automatically created 3D volume data sets. All volumes were analyzed using the built-in 3D software program, and images were recorded. Volume-rendered, maximum intensity projection and multiplanar reconstruction algorithms were used to examine the luminal surface and plaque echo-structure. In 3D software programs, the whole surface of the carotid plaque was examined from different angles, while displaying it on the monitor. The 3D sonographic examination and image reconstruction procedures were completed within 3 to $5 \mathrm{~min}$, for each patient. One month later after collecting all 2D and 3D US data, two independent observers, each blinded to the results of the other, reviewed all images separately.

\section{Statistical analysis}

All 2D and 3D sonographic findings were compared. Statistical analysis was performed with 
SPSS version 12.0 software (SPSS Inc, Chicago, IL). The Kolmogorov-Smirnov test was used to check the normality of the variables' distributions. The Mann-Whitney $U$ test was used to compare these results. In all statistical analyses a significance level of $p<0.05$ was used. The $\kappa$ coefficient was used for inter-observer variability, with a $\kappa<0.20$ defined as poor inter-observer agreement; 0.21 to 0.40 as fair; 0.41 to 0.60 as moderate; 0.61 to 0.80 as good; and 0.81 to 1.00 as very good, or almost perfect.

\section{Results}

The clinical characteristics of the patients are presented in Table I. Significant differences were found between male and female cases for hypertension, diabetes mellitus, and tobacco use, and no significant differences were found for other clinical characteristics. The class distribution of carotid plaques was homogeneous.

Three-dimensional imaging was successfully performed in 45 of the 50 cases and the technical success was $90 \%$. In 5 of the cases, 3D volumes could not be evaluated properly because of a reverberation artifact in two cases (in near wall located carotid plaques, optimal images cannot be obtained because of the near wall that causes sonographic reverberation artifacts) and patient incompliance in 3 other cases (short patient neck and high carotid bifurcation), similarly as in the literature [14]. The plaques ranged from 2 to $12 \mathrm{~mm}$ in length (mean: $3.98 \pm 1.70 \mathrm{~mm}$ ) and from 1.8 to $3.2 \mathrm{~mm}$ in width (mean: $2.11 \pm 0.37 \mathrm{~mm}$ ). All patients had asymptomatic low-grade carotid stenosis.

Of the 45 evaluated patients, 3 (6.7\%) plaques were located in the common carotid artery, $25(55.5 \%)$ plaques on the carotid bulb, and 17 (37.8\%) plaques on the internal carotid arteries. The sites of the plaques were as follows: on the far wall in $35(77.8 \%)$ cases, eccentrically located in $8(17.8 \%)$ cases and on the near vessel wall in $2(4.4 \%)$ cases. A single plaque was detected in $64.4 \%$ of cases, with the remaining $35.6 \%$ of the patients having multiple plaques. No ulcerated plaques were observed in either the $2 \mathrm{D}$ or the $3 \mathrm{D}$ images.

There were no significant differences between 2D and 3D US assessment results for carotid plaque echo-structures (observer 1, $p=0.317$; observer 2, $p=0.276$ ). The inter-observer agreement on 2D and 3D US for plaque echo-structure was very good, and the k coefficients of inter-observer agreement were acceptable (0.89 and 0.83 , respectively). Table II shows the $2 \mathrm{D}$ and $3 \mathrm{D}$ US results of assessment for carotid plaque echo-structures.

There were significant differences between 2D and 3D plaque surface irregularity (observer 1 , $p=0.002$; observer $2, p=0.004$ ) (Figures $1-5$ ). The ratio of plaque irregularity observed in $3 \mathrm{D}$ evaluations was higher. The inter-observer agreement on 2D and 3D US for surface irregularity was very good, and the $k$ coefficients of inter-observer agreement on 2D and 3D plaque surface irregularity were also acceptable (0.91 and 0.95 , respectively). Table III presents the 2D and 3D US results for plaque surface regularity.

\section{Discussion}

The results of this study suggest that 3D US examinations are superior to 2D US examination for assessment of plaque surface irregularity and as a non-invasive assessment provides a practical way to emphasize the whole plaque surface. We found a higher rate of irregularity with 3D than with 2D US investigations, consistent with previous data, and there was a good agreement in the evaluation of surface irregularity with the 3D US technique $(\kappa=0.95)$. In addition, evaluation

Table I. Clinical characteristics of the study population

\begin{tabular}{|c|c|c|c|}
\hline Variable & Women $(n=20)$ & Men $(n=30)$ & Total $(n=50)$ \\
\hline Age [years] & $67.6 \pm 7.2$ & $69.6 \pm 5.6$ & $68.5 \pm 7.8$ \\
\hline BMI $\left[\mathrm{kg} / \mathrm{m}^{2}\right]$ & $29.6 \pm 5.1$ & $28.2 \pm 5.4$ & $29.2 \pm 5.2$ \\
\hline Hypertension & $8(40 \%)$ & $19(63.3 \%)$ & 27 (54\%) \\
\hline Diabetes mellitus & $6(30 \%)$ & $11(36.7 \%)$ & $17(34 \%)$ \\
\hline Former tobacco use & $1(5 \%)$ & $14(47.7 \%)$ & $15(30 \%)$ \\
\hline Current tobacco use & $1(5 \%)$ & $9(30 \%)$ & $10(20 \%)$ \\
\hline High-density lipoprotein $[\mathrm{mg} / \mathrm{dl}]^{*}$ & $52.1 \pm 10.2$ & $54.4 \pm 19.4$ & $53.4 \pm 16.7$ \\
\hline Low-density lipoprotein [mg/dl] & $142.13 \pm 18.8$ & $146 \pm 12.4$ & $145.6 \pm 19.9$ \\
\hline History of cardiovascular disease & $4(20 \%)$ & $7(23.3 \%)$ & $11(22 \%)$ \\
\hline
\end{tabular}

${ }^{\star}$ Data are mean \pm standard deviation. 
Table II. The 2D and 3D ultrasonographic findings in relation to plaque echo-structure. Class I: homogeneous hypoechoic; class II: heterogeneous predominantly hypoechoic; class III: heterogeneous predominantly hyperechoic; class IV: homogeneous hyperechoic, and class V: calcified plaque

\begin{tabular}{|c|c|c|c|c|c|c|c|}
\hline \multirow[t]{2}{*}{ Variable } & \multicolumn{7}{|c|}{ Plaque echo-structure } \\
\hline & $\begin{array}{l}\text { Class I } \\
n(\%)\end{array}$ & $\begin{array}{c}\text { Class II } \\
n(\%)\end{array}$ & $\begin{array}{c}\text { Class III } \\
n(\%)\end{array}$ & $\begin{array}{c}\text { Class IV } \\
n(\%)\end{array}$ & $\begin{array}{c}\text { Class V } \\
n(\%)\end{array}$ & Total & $\begin{array}{c}\text { Inter-observer } \\
\text { agreement } \\
\text { ( } \kappa \text { value) }\end{array}$ \\
\hline 2D US: & & & & & & & 0.89 \\
\hline Observer 1 & $7(15.6 \%)$ & $4(8.9 \%)$ & $11(24.4 \%)$ & $13(28.9 \%)$ & $10(22.2 \%)$ & 45 & \\
\hline Observer 2 & $6(13.3 \%)$ & $4(8.9 \%)$ & $11(24.4 \%)$ & $12(26.7 \%)$ & $12(26.7 \%)$ & 45 & \\
\hline 3D: & & & & & & & 0.83 \\
\hline Observer 1 & 7 (15.6\%) & 4 (8.9\%) & 11 (24.4\%) & 12 (26.7\%) & 11 (24.4\%) & 45 & \\
\hline Observer 2 & 6 (13.3\%) & $4(8.9 \%)$ & 9 (20.0\%) & 13 (28.9\%) & 13 (28.9\%) & 45 & \\
\hline
\end{tabular}

of 3D sonographic images, with respect to surface regularity, may be more objective and easier than evaluating 2D sonographic images.

In the Cardiovascular Health Study (CHS), it was reported that $75 \%$ of men and $62 \%$ of females over 64 years of age had detectable carotid artery stenosis, with most of these cases at a low level of stenosis [15]. Among those with asymp- tomatic carotid stenosis, the annual risk of CVDs is estimated to be from $1.3 \%$ to $3.3 \%$ [16].

Ultrasonographic echo-structure of the atheroma plaque is an important well-known risk factor in CVDs, with hypoechoic plaques being unstable and constituting a high risk factor for CVDs [10, $17,18]$. Recent studies have reported that a positive correlation between plaque echolucency and
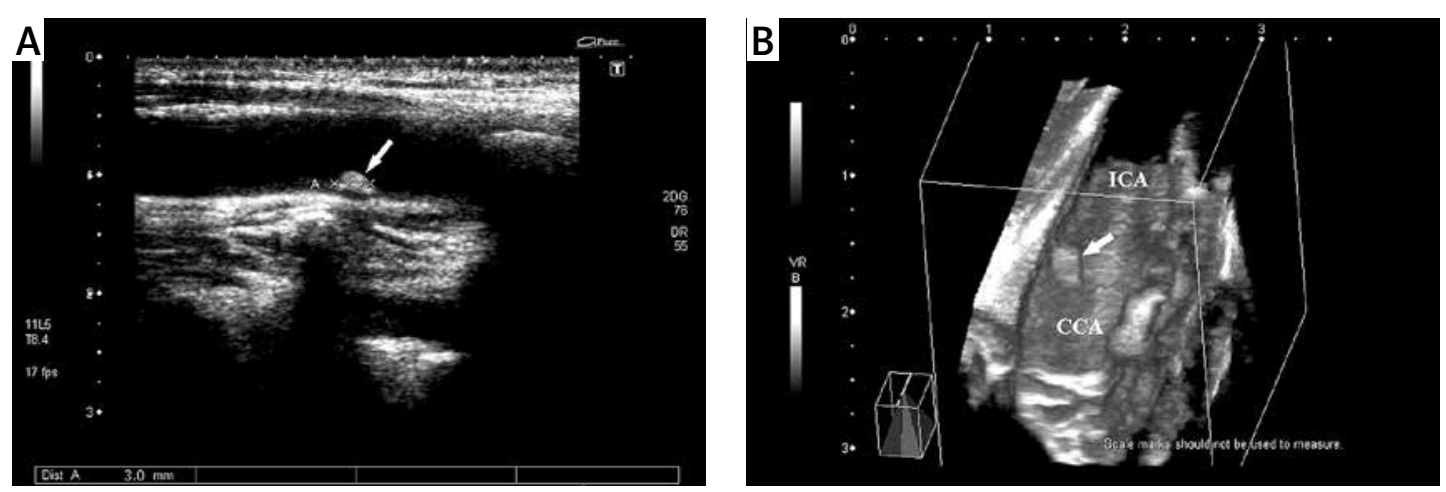

Figure 1. Fifty-nine-year-old man with asymptomatic carotid artery plaque. A - A hyperechogenic type IV atheroma plaque (arrow) (with acoustic shadowing artifact) with regular surface is observed in the carotid bulb on 2D. B - 3D ultrasonographic image

ICA - internal carotid artery, CCA - common carotid artery.
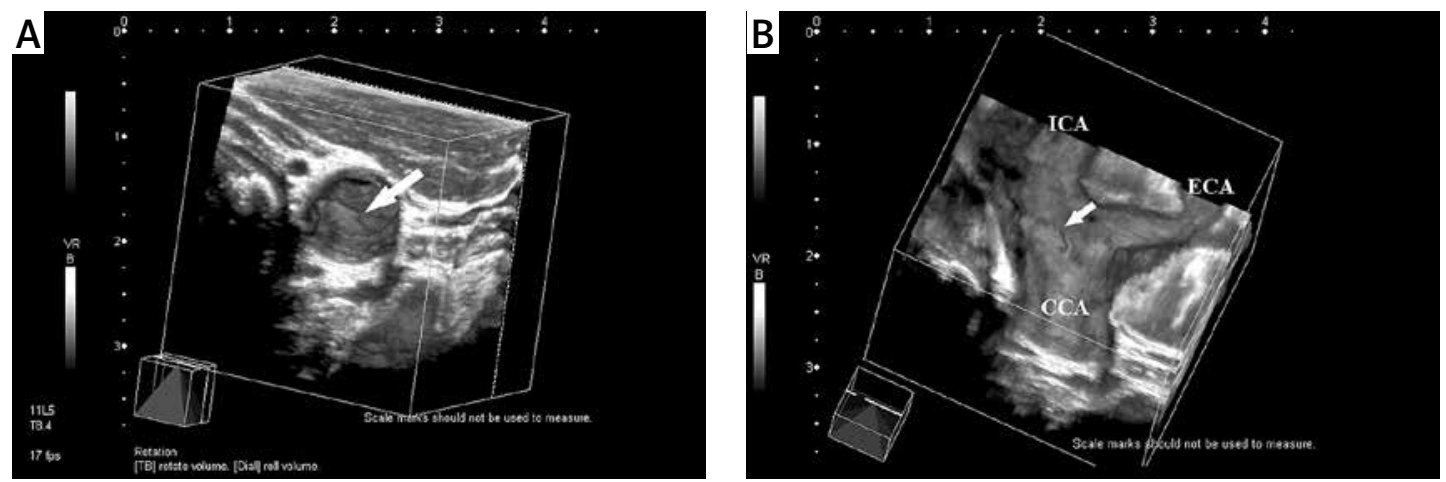

Figure 2. Sixty-one-year-old woman with asymptomatic carotid artery plaque. A - View of a type III, heterogeneous hyperechogenic atheroma plaque (arrow) with an irregular surface, located in the carotid bulb, as seen from the distal common carotid artery on the three-dimensional ultrasonographic image; B - plaque (arrow) with irregular surface in the carotid bulb and internal carotid artery are seen on the image obtained after volume sectioning procedure

ICA - internal carotid artery, CCA - common carotid artery, ECA - external carotid artery. 

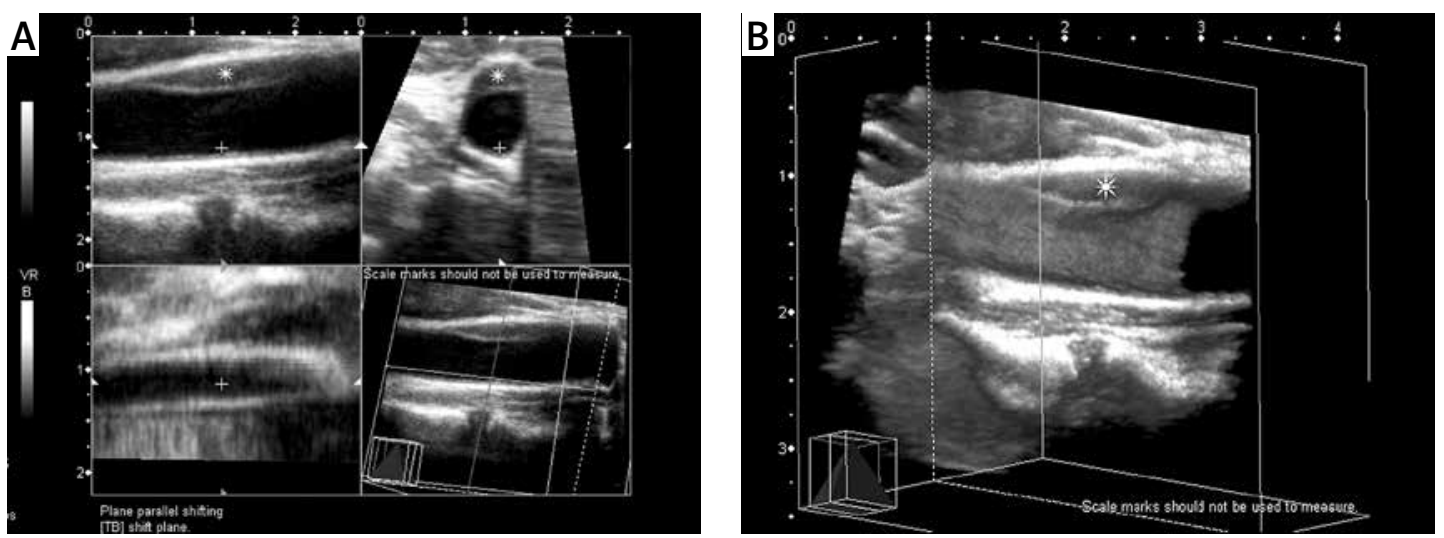

Figure 3. Seventy-year-old man with asymptomatic carotid artery disease. A - A hypoechogenic type I atheroma plaque (asterisk) with a regular surface is observed in the common carotid artery on the multiplanar imaging; B - 3D ultrasonographic image
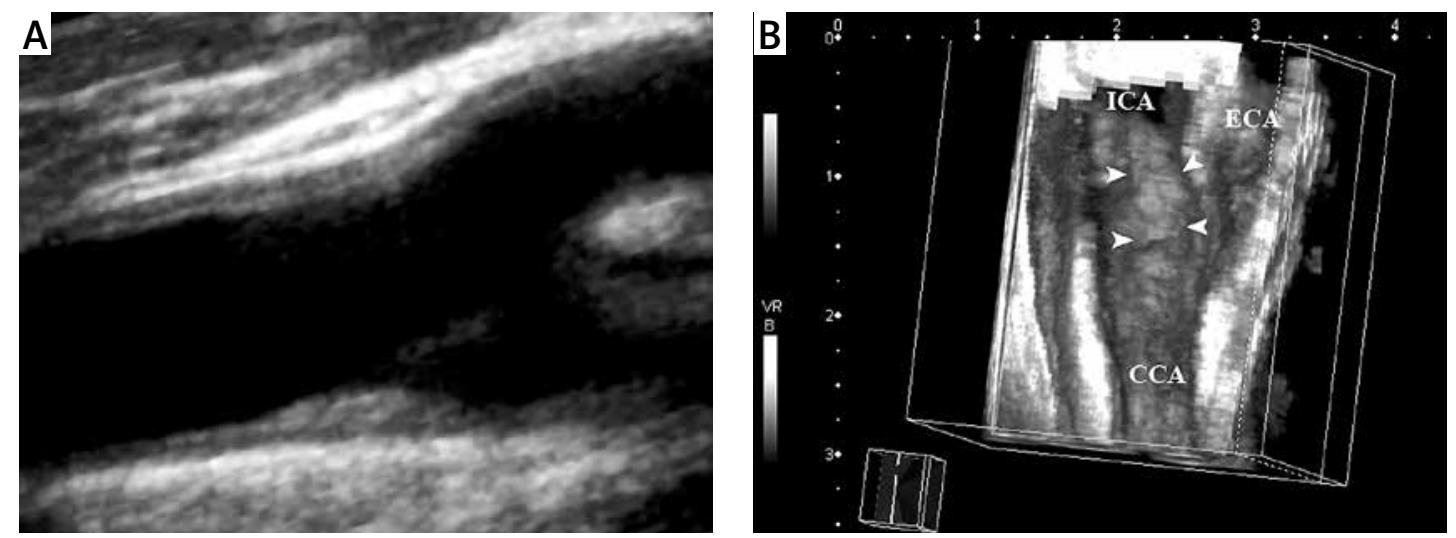

Figure 4. Seventy-one-year-old woman with asymptomatic carotid artery plaque. A - Hypoechogenic type II atheroma plaque with a smooth surface on the far wall of the common carotid artery on 2D ultrasonographic examination (longitudinal view); B - however, on 3D ultrasonographic imaging, an irregular plaque surface is observed (arrowheads)
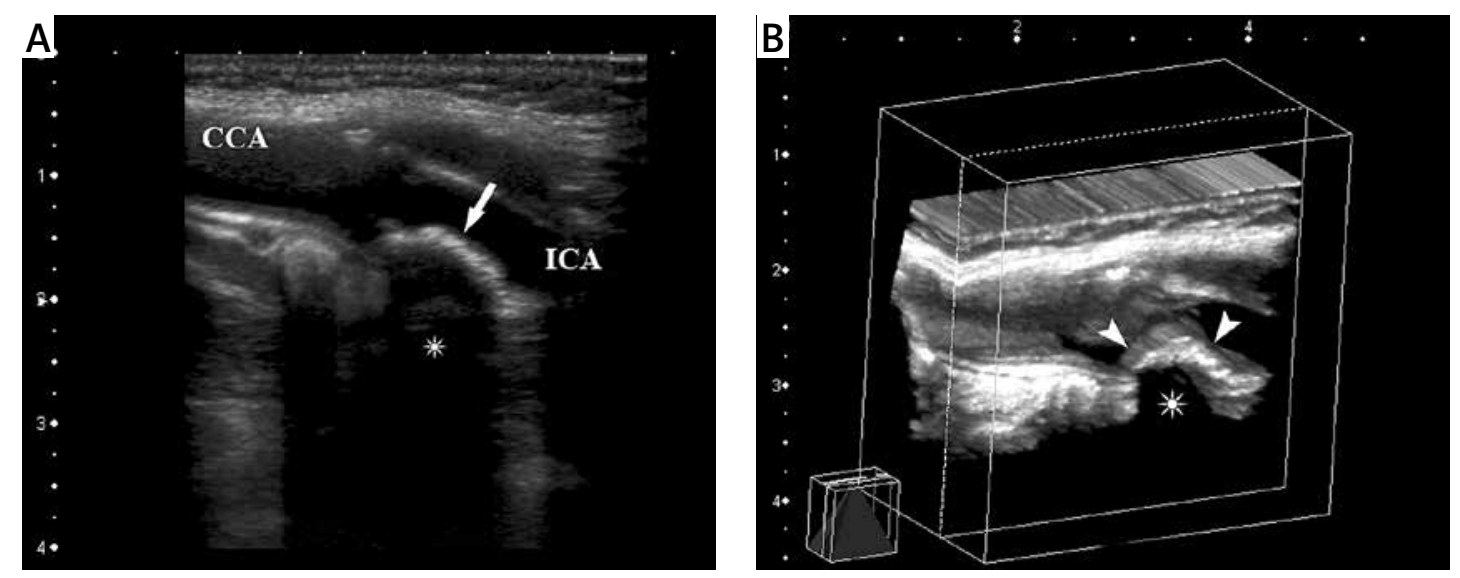

Figure 5. Seventy-six-year-old man with asymptomatic carotid artery plaque. A - Calcified type $V$ plaque (arrow) with a smooth surface on the far wall of the internal carotid artery is observed on 2D ultrasonographic (longitudinal view); B - 3D virtual arterioscopic image (arrowheads). Calcified plaques are observed on both images with posterior acoustic shadowing (asterisk)

cerebral ischemic events was determined in patients with a $35 \%$ to $50 \%$ carotid artery stenosis $[10,19]$.

Clinicopathologic studies have reported that irregular or ulcerated plaque surface morphology has also been correlated with a higher degree of stenosis, and also with ischemic CVDs [3, 7, 20]. These data suggest that plaque surface irregularity, even after adjusting for the degree of stenosis, is an independent predictor of ischemic CVDs [7, 20, 21]. 
Table III. The 2D and 3D ultrasonographic findings in relation to plaque surface regularity

\begin{tabular}{|c|c|c|c|c|}
\hline \multirow[t]{2}{*}{ Variable } & \multicolumn{4}{|c|}{ Plaque regularity } \\
\hline & $\begin{array}{l}\text { Smooth } \\
n(\%)\end{array}$ & $\begin{array}{l}\text { Irregular } \\
n(\%)\end{array}$ & Total & $\begin{array}{c}\text { Inter-observer } \\
\text { agreement ( } \kappa \text { value) }\end{array}$ \\
\hline 2D US: & & & & 0.91 \\
\hline Observer 1 & 38 (84.4\%) & 7 (15.6\%) & 45 & \\
\hline Observer 2 & 39 (86.7\%) & $6(13.3 \%)$ & 45 & \\
\hline 3D US: & & & & 0.95 \\
\hline Observer 1 & $28(62.2 \%)$ & $17(37.8 \%)$ & 45 & \\
\hline Observer 2 & $29(64.4 \%)$ & 16 (35.6\%) & 45 & \\
\hline
\end{tabular}

Also, Prati et al. [21] reported that plaque surface irregularity, echolucency and texture characteristics, compounded in a plaque score, might be independent predictors of the occurrence of cerebrovascular ischemic events in a general population, independently of the stenosis degree evaluation. Kanber et al. [22] reported that an objective assessment of plaque surface irregularities using a novel surface irregularity index may correlate with the presence of ipsilateral hemispheric cerebrovascular symptoms. They found an increase in diagnostic performance using the plaque surface irregularity versus that provided by the degree of stenosis alone. Therefore plaque surface irregularity may be a valuable tool for improving risk assessment and can be helpful to identify vulnerable plaques in patients with carotid artery disease [22, 23].

As a result of this information, detecting carotid artery plaques and characterizing their echo-structures and luminal surfaces have become a more important part of the clinical follow-up. Conventional angiography in evaluating patients who have carotid arteries is the most commonly used method and remains the gold standard for screening patients and determining which patients should receive an interventional therapy [3]. However, carotid angiography is an invasive method, with a morbidity rate of $1 \%$ to $4 \%$ and a $1 \%$ risk of peri-interventional CVDs [9]. In addition, conventional angiography only shows the free lumen. It does not provide information about the plaque structure, the arterial walls, or the true arterial lumen [3]. Magnetic resonance (MR) and computed tomography (CT) angiography are other screening methods used in carotid artery imaging. However, both of these are relatively expensive and not easily accessible, they take a long time to examine and they require the administration of contrast media.

Currently, the preferred non-invasive method for detection and follow-up of plaques is 2D US. In addition to providing an opportunity to measure various parameters of carotid artery plaques, such as the degree of stenosis, surface regularity, echo-structure and intima-media thickness, 2D
US can evaluate plaque regression or progression. However, 2D US examination is operator-dependent and has limitations. The selected images are often not representative of the composition of the entire plaque. Also, the atherosclerotic plaque often develops asymmetrically. As a result, the 2D method requires a subjective reconstruction of the plaque shape [24-27].

Several studies have reported that the 3D analysis method allows more precise characterization of the surface regularity, the echo-structure of the carotid plaque and the volume measurement of the plaque [10, 24-34]. Previous studies aimed at evaluating the reproducibility of 3D ultrasound in the volume measurement of carotid plaque showed good intra- and inter-observer agreement ( $\kappa$ coefficients ranging from $0.94-0.96$ to $0.89-$ 0.93 , respectively) $[28,31,32]$. The inter-observer agreement in the reproducibility of 3D ultrasound in carotid plaque irregularity and ulceration was also good ( $\kappa$ coefficients, 0.90 and 0.97 respectively) in two recent studies [29, 33]. In addition, fine intra- and inter-observer reproducibility was reported in evaluating the reproducibility of 3D ultrasound in carotid plaque echo-structures, with intra- and inter-observer correlation coefficients of $>0.93$ and $>0.89$, respectively $[10$, 34]. Three-dimensional US investigations provide good intra- and inter-observer agreement at the same high accuracy rate as in an objective method. Also, Johri et al. [35] recently reported that 3D ultrasound-based carotid plaque quantification may serve as an important clinical screening tool to help identify patients who are at low risk for significant coronary artery disease.

The number of patients is relatively small. Also, because all of our patients were asymptomatic, we did not include catheter angiography, CT-MR angiography studies, or pathologic samples in this study. These could have helped in comparing carotid artery plaques detected in the patients. Three-dimensional US data were obtained using a free hand technique. The mechanical scanning technique is an expensive and impractical method 
in routine use. The free hand technique is practical, cheap and reproducible in routine use. With the free-hand technique, there might be gaps between the two-dimensional reference images and distortions may occur in secondary images, depending on the scanning speed. If the examiners perform 3D scanning at a fixed and suitable velocity, these limitations can be easily avoided. Moreover, movement artifacts related to swallowing and respiration may occur [30].

In conclusion, besides the stenosis degree, plaque echo-structure and surface irregularity are important in determining the risk of CVDs in carotid artery disease. Especially in the asymptomatic group, demonstration of carotid plaque surface irregularity with non-invasive imaging methods is very important in prevention and treatment of carotid artery stenosis and cerebrovascular events and follow-up. In this study, we observed that 3DUS technique is superior to 2D-US in demonstrating luminal surface irregularity. Further studies with a large number of patients are necessary to investigate our hypothesis.

\section{Conflict of interest}

The authors declare no conflict of interest.

\section{References}

1. Eliasziw M, Streifler JY, Fox AJ, Hachinski VC, Ferguson GG, Barnett HJ. Significance of plaque ulceration in symp tomatic patients with high-grade carotid stenosis. North American Symptomatic Carotid Endarterectomy Trial. Stroke 1994; 25: 304-8.

2. Inzitari D, Eliasziw M, Gates P, et al. The causes and risk of stroke in patients with asymptomatic internal-carotid-artery stenosis. N Engl J Med 2000; 342: 1693-700.

3. Rothwell PM, Gibson R, Warlow CP. Interrelation between plaque surface morphology and degree of stenosis on carotid angiograms and the risk of ischemic stroke in patients with symptomatic carotid stenosis. On behalf of the European Carotid Surgery Trialists' Collaborative Group. Stroke 2000; 31: 615-21.

4. Rothwell PM, Eliasziw M, Gutnikov SA, et al. Carotid Endarterectomy Trialists' Collaboration. Analysis of pooled data from the randomised controlled trials of endarterectomy for symptomatic carotid stenosis. Lancet 2003; 361: 107-16.

5. Brott TG, Hobson RW $2^{\text {nd }}$, Howard G, et al. Stenting versus endarterectomy for treatment of carotid-artery stenosis. N Engl J Med 2010; 363: 11-23.

6. North American Symptomatic Carotid Endarterectomy Trial Collaborators. Beneficial effect of carotid endarterectomy in symptomatic patients with high-grade carotid stenosis. North American Symptomatic Carotid Endarterectomy Trial Collaborators. N Engl J Med 1991; 325: 445-53.

7. Prabhakaran S, Rundek T, Ramas R, et al. Carotid plaque surface irregularity predicts ischemic stroke: the Northern Manhattan Study. Stroke 2006; 37: 2696-701.

8. Lal BK. Sonographic evaluation in carotid artery stenosis. In: Imaging of Carotid Artery Stenosis. Schaller BJ (ed.). Springer Wien, New York 2007; 35-9.
9. Endarterectomy for asymptomatic carotid stenosis. Executive Committee for the Asymptomatic Carotid Atherosclerosis Study. JAMA 1995; 273: 1421-8.

10. Heliopoulos J, Vadikolias K, Mitsias P, et al. A three-dimensional ultrasonographic quantitative analysis of nonulcerated carotid plaque morphology in symptomatic and asymptomatic carotid stenosis. Atherosclerosis 2008; 198: 129-35.

11. de Bray JM, Baud JM, Dauzat M. Consensus concerning the morphology and the risk of carotid plaques. Cerebrovasc Dis 1997; 7: 289-96.

12. Touboul PJ, Labreuche J, Vicaut E, Amarenco P; GENIC Investigators. Carotid intima-media thickness, plaques, and Framingham risk score as independent determinants of stroke risk. Stroke 2005; 36: 1741-5.

13. Polak JF, O'Leary DH, Kronmal RA, et al. Sonographic evaluation of carotid artery atherosclerosis in the elderly: relationship of disease severity to stroke and transient ischemic attack. Radiology 1993; 188: 363-70.

14. Vicenzini E, Galloni L, Ricciardi MC, et al. Advantages and pitfalls of three-dimensional ultrasound imaging of carotid bifurcation. Eur Neurol 2011; 65: 309-16.

15. O'Leary DH, Polak JF, Kronmal RA, et al. Distribution and correlates of sonographically detected carotid artery disease in the Cardiovascular Health Study. The CHS Collaborative Research Group. Stroke 1992; 23: 1752-60.

16. Norris JW, Zhu CZ, Bornstein NM, Chambers BR. Vascular risks of asymptomatic carotid stenosis. Stroke 1991; 22: 1485-90.

17. Reiter M, Effenberger I, Sabeti S, et al. Increasing carotid plaque echolucency is predictive of cardiovascular events in high-risk patients. Radiology 2008; 248: 1050-5.

18. Mathiesen EB, Bonaa KH, Joakimsen O. Echolucent plaques are associated with high risk of ischemic cerebrovascular events in carotid stenosis: the Tromso study. Circulation 2001; 103: 2171-5.

19. Kitamura A, Iso H, Imano H, et al. Carotid intima-media thickness and plaque characteristics as a risk factor for stroke in Japanese elderly men. Stroke 2004; 35: 2788-94.

20. Rosenkranz M, Russjan A, Goebell E, et al. Carotid plaque surface irregularity predicts cerebral embolism during carotid artery stenting. Cerebrovasc Dis 2011; 32: 163-9.

21. Prati P, Tosetto A, Casaroli M, et al. Carotid plaque morphology improves stroke risk prediction: usefulness of a new ultrasonographic score. Cerebrovasc Dis 2011; 31: 300-4.

22. Kanber B, Hartshorne TC, Horsfield MA, Naylor AR, Robinson TG, Ramnarine KV. Quantitative assessment of carotid plaque surface irregularities and correlation to cerebrovascular symptoms. Cardiovasc Ultrasound 2013; 11: 38

23. Ibrahimi $P$, Jashari $F$, Johansson $E$, et al. Vulnerable plaques in the contralateral carotid arteries in symptomatic patients: a detailed ultrasound analysis. Atherosclerosis 2014; 235: 526-31.

24. Meairs S, Beyer J, Hennerici M. Reconstruction and visualization of irregularly sampled three- and four-dimensional ultrasound data for cerebrovascular applications. Ultrasound Med Biol 2000; 26: 263-72.

25. Perez HA, Garcia NH, Spence JD, Armando LJ. Adding carotid total plaque area to the Framingham risk score improves cardiovascular risk classification. Arch Med Sci 2016; 12: 513-20.

26. Cheng HG, Patel BS, Martin SS, et al. Effect of comprehensive cardiovascular disease risk management on 
longitudinal changes in carotid rtery intima-media thickness in a community-based prevention clinic. Arch Med Sci 2016; 12: 728-35.

27. Barry CD, Allott CP, John NW, et al. Three-dimensional freehand ultrasound: image reconstruction and volume analysis. Ultrasound Med Biol 1997; 23: 1209-24.

28. Landry A, Spence JD, Fenster A. Measurement of carotid plaque volume by 3 -dimensional ultrasound. Stroke 2004; 35: 864-9.

29. Schiminke U, Motsch L, Hilker L, Kessler C. Three-dimensional ultrasound observation of carotid artery plaque ulceration. Stroke 2000; 31: 1651-5.

30. Fenster A, Blake C, Gyacskov I, Landry A, Spence JD. 3D ultrasound analysis of carotid plaque volume and surface morphology. Ultrasonics 2006; 44: 153-7.

31. Pollex RL, Spence JD, House AA, et al. A comparison of ultrasound measurements to assess carotid atherosclerosis development in subjects with and without type 2 diabetes. Cardiovasc Ultrasound 2005; 3: 15.

32. Griewing B, Schminke U, Morgenstern C, Walker ML, Kessler C. Three-dimensional ultrasound angiography (power mode) for the quantification of carotid artery atherosclerosis. J Neuroimaging 1997; 7: 40-5.

33. Heliopoulos J, Vadikolias K, Piperidou C, Mitsias P. Detection of carotid artery plaque ulceration using 3-dimensional ultrasound. J Neuroimaging 2011; 21: 126-31.

34. Denzel C, Balzer K, Merhof D, Lang W. 3D cross sectional view to investigate the morphology of internal carotid artery plaques. Is 3D ultrasound superior to 2D ultrasound? Ultraschall Med 2009; 30: 291-6.

35. Johri AM, Chitty DW, Matangi M, et al. Can carotid bulb plaque assessment rule out significant coronary artery disease? A comparison of plaque quantification by twoand three-dimensional ultrasound. J Am Soc Echocardiogr 2013; 26: 86-95. 